\title{
Fertilización fosfatada para lechuga en un Oxisol con altos contenidos de fósforo disponible
}

\section{Phosphate fertilization for lettuce in oxisol with high available phosphorus content}

\section{Arthur Bernardes CECíLIO FILHO ${ }^{1}$; Giovani Donizete BONELA²; Mara Cristina Pessoa da CRUZ $^{3}$; Sergio Manuel RUGELES-REYES ${ }^{4}$; Ana Carolina Foresti MENEZELLO ${ }^{5}$}

\author{
1 Professor Dr. Universidade Estadual Paulista "Júlio de Mesquita Filho" - Unesp, Faculdade de Ciências Agrarias e \\ Veterinárias, campus de Jaboticabal; rutra@fcav.unesp.br \\ ${ }^{2}$ Professor M.Sc. Universidade Estadual de Goias; giovanibonela@bol.com.br \\ 3 Professora Dr. Universidade Estadual Paulista "Júlio de Mesquita Filho" - Unesp, Faculdade de Ciências Agrarias e \\ Veterinárias, campus de Jaboticabal; mcpcruz@fcav.unesp.br \\ ${ }^{4}$ Autor para correspondência: Eng. Agr., mestrando em Agronomia; Universidade Estadual Paulista “Júlio de Mesquita Filho" - \\ Unesp, Faculdade de Ciências Agrarias e Veterinárias, campus de Jaboticabal; smrugelesr@gmail.com \\ ${ }^{5}$ Eng. Agr., Universidade Estadual Paulista "Júlio de Mesquita Filho" - Unesp, Faculdade de Ciências Agrarias e Veterinárias, \\ campus de Jaboticabal; rutra@fcav.unesp
}

Recebido em: 14-06-2017; Aceito em: 03-10-2017

\begin{abstract}
Resumen
El cultivo intensivo de hortalizas en la misma área puede provocar aumento del contenido de fósforo en el suelo, traduciéndose a veces en prejuicios para el medio ambiente y la planta. El objetivo de este estudio fue evaluar la respuesta de tres cultivares de lechuga, 'Amanda' (grupo de hojas crespas y sueltas), 'Karla' (grupo de hojas lisas y sueltas) y 'Lucy Brown' (grupo de hojas crespas con formación de cabeza) a cinco dosis de fósforo $\left(0,50,100,200\right.$ y $300 \mathrm{~kg} \mathrm{ha}^{-1}$ de $\left.\mathrm{P}_{2} \mathrm{O}_{5}\right)$, en un Oxisol con alto contenido de este nutriente (136 $\mathrm{mg} \mathrm{dm}^{-3}$ ), en un diseño experimental de bloques aleatorizados, arreglo factorial $3 \times 5$ con cuatro repeticiones. Se encontró interacción significativa de los factores para concentración foliar de P. Mayores valores de masa fresca y seca fueron observados en 'Lucy Brown', además, en todos los cultivares se encontró aumento con el incremento de $\mathrm{P}$, al igual que en la concentración foliar de este elemento. El número de hojas $(\mathrm{NH})$ y el área foliar $(\mathrm{AF})$ no fueron influenciados por la interacción, pero sí por la dosis, ajustándose a una ecuación cuadrática, siendo 161 y $203 \mathrm{~kg} \mathrm{ha}^{-1}$ de $\mathrm{P}_{2} \mathrm{O}_{5}$ las dosis que proporcionaron mayores $\mathrm{NH}$ y AF, respectivamente. La fertilización fosfatada promovió incrementos significativos para todas las características de lechuga hasta la dosis de $300 \mathrm{~kg} \mathrm{ha}^{-1}$ de $\mathrm{P}_{2} \mathrm{O}_{5}$.
\end{abstract}

Palabras clave adicionales: cultivares, Lactuca sativa, nutrición mineral.

\section{Resumo}

O cultivo intensivo de hortaliças na mesma área pode provocar aumento do teor de fósforo no solo, causando, às vezes, prejuízos para o meio ambiente e à planta. $O$ objetivo deste trabalho foi avaliar a resposta de três cultivares de alface, 'Amanda' (grupo de folhas crespas e soltas), 'Karla' (grupo de folhas lisas e soltas) e 'Lucy Brown' (grupo de folhas crespas com formação de cabeça) à cinco doses de fósforo $(0,50,100$, 200 e $300 \mathrm{~kg} \mathrm{ha}^{-1}$ de $\left.\mathrm{P}_{2} \mathrm{O}_{5}\right)$, em um Latossolo com alto teor deste nutriente $\left(136 \mathrm{mg} \mathrm{dm}^{-3}\right)$, em blocos ao acaso, com arranjo dos tratamentos em fatorial $3 \times 5$, e quatro repetições. Observou-se interação significativa dos fatores para teor foliar de P. Maiores valores de massa fresca e seca foram observados em 'Lucy Brown'; porém, em todas as cultivares foram observados aumentos com o incremento de P aplicado, assim como também aumento do teor foliar deste nutriente. Não houve interação dos fatores no número de folhas $(\mathrm{NH})$ e na área foliar (AF), mas estas características foram afetadas pela dose, ajustando-se à equações quadráticas, sendo 161 e $203 \mathrm{~kg} \mathrm{ha}^{-1}$ de $\mathrm{P}_{2} \mathrm{O}_{5}$ as doses que proporcionaram maiores $\mathrm{NH}$ e AF, respectivamente. A fertilização fosfatada promoveu incrementos significativos para todas as características de alface até a dose de $300 \mathrm{~kg} \mathrm{ha}^{-1}$ de $\mathrm{P}_{2} \mathrm{O}_{5}$.

Palavras-chave adicionais: cultivares, Lactuca sativa, nutrição mineral.

\section{Summary}

The intensive cultivation of lettuce in the same area may cause an increase in phosphorus in the soil, causing damage to the environment and plants. The objective of this study was to evaluate the response of three cultivars of lettuce, 'Amanda' (crisp and loose leaves group), 'Karla' (smooth and loose leaves group) and 'Lucy Brown' (crisphead group) and five doses of phosphorus $\left(0,50,100,200\right.$ and $300 \mathrm{~kg} \mathrm{ha}^{-1}$ of $\mathrm{P}_{2} \mathrm{O}_{5}$ ) in an Oxisol with high levels of this nutrient $\left(136 \mathrm{mg} \mathrm{dm}^{-3}\right)$ in a randomized block design in $3 \times 5$ factorial experi- 
ment with four replications. Statistical analysis was significant for interaction of factors on the P foliar content. Higher values of fresh and dry weight were observed for 'Lucy Brown'; furthermore, all the cultivars had a linear increase with $\mathrm{P}$ enhancement, as noticed for $\mathrm{P}$ content. The number of leaves $(\mathrm{NH})$ and leaf area $(\mathrm{AF})$ were not influenced by the interaction and set up the quadratic equation where a dose of 161 and $203 \mathrm{~kg} \mathrm{ha}^{-1}$ $\mathrm{P}_{2} \mathrm{O}_{5}$ provided higher $\mathrm{NH}$ and $\mathrm{AF}$, respectively. Phosphorus fertilization promoted significant increases for all lettuce characteristics up to the dose of $300 \mathrm{~kg} \mathrm{ha}^{-1} \mathrm{P}_{2} \mathrm{O}_{5}$.

Additional keywords: cultivars, Lactuca sativa, mineral nutrition.

\section{Introducción}

La lechuga es la hortaliza más cultivada en Brasil (Petrazzini et al., 2014). El consumo generalmente es in natura, y viene intensificándose en los últimos años como consecuencia del crecimiento poblacional y por sus características nutricionales como fuente de sales minerales y vitaminas, además del bajo contenido de calorías (Batista et al., 2012). En el estado de São Paulo, el grupo predominante es el de la lechuga crespa seguida de la americana y lisa (Sala \& Costa, 2012).

La lechuga es considerada una planta exigente en nutrientes (Mantovani et al., 2014), en la cual, las cantidades de fósforo acumuladas son bajas en comparación a las de potasio y nitrógeno (Kano et al., 2012), sin embargo, muestra respuesta positiva en producción y calidad a la aplicación del macronutriente (AlKhader \& Abu Rayyan, 2015).

Dependiendo del tipo de suelo y de la disponibilidad de $\mathrm{P}$, para lechuga se recomienda aplicación de 200 a $400 \mathrm{~kg} \mathrm{ha}^{-1}$ de $\mathrm{P}_{2} \mathrm{O}_{5}$ (Trani et al., 1997). No obstante, esta recomendación no considera el cultivar o grupo al que pertenece la lechuga. En la literatura, se encuentra divergencia de la dosis óptima para proporcionar máxima producción (Kano et al., 2012; Bertossi et al., 2013; Mantovani et al., 2014; Silveira et al., 2015). Además, de acuerdo a Cecílio Filho et al. (2015), la mayoría de productores no toma en consideración el análisis de suelo, aplicando grandes cantidades de fertilizantes. Esto, sumado al hecho que la planta posee ciclo corto y se hacen múltiples cultivos a lo largo del año, resulta en elevados contenidos de $P$ en el suelo (Mantovani et al., 2014).

Según Silva et al. (2016), aplicaciones eleva- das de fertilizantes han tenido impacto positivo para alcanzar y mantener los rendimientos de los cultivos, sin embargo, las grandes cantidades de $P$ en los suelos pueden traer consecuencias negativas para el ambiente, como la acumulación y contaminación de suelos por metales pesados (Jiao et al., 2012) y la eutrofización de manantiales y cuerpos de agua, la cual ya es reportada como un problema ambiental en diferentes regiones del mundo (Tóth et al., 2014). Igualmente, la oferta mundial de $\mathrm{P}$ es finita y no renovable (Jordan-Meille et al., 2012). De esta manera, el uso de fertilizantes con $P$ debe ser estudiado para garantizar un ambiente sustentable y la mejor utilización posible por los cultivos.

Teniendo en cuenta lo anterior y considerando que la mayoría de estudios que evalúan la respuesta de la lechuga a fertilización fosfatada han sido realizados en suelos con bajo contenido de $\mathrm{P}$ (Coutinho et al., 2008; Silva, 2013; Mantovani et al., 2014), el objetivo de este estudio fue evaluar la respuesta de lechuga a dosis de fósforo, en un Oxisol con altos contenidos del nutriente.

\section{Materiales y métodos}

\section{Localización y caracterización del área experimental}

El experimento fue realizado en el periodo de 5 de mayo a 31 de julio de 2008 en la Unesp, Campus de Jaboticabal, localizado en el municipio de Jaboticabal, estado de São Paulo, a 2115'22" Sur, 48¹8'58" Oeste, y altitud de 575 metros. Los valores de temperaturas mínimas, máximas y precipitación referentes al periodo del experimento se encuentran en la Tabla 1.

Tabla 1- Datos meteorológicos del período experimental. Meteorological data of the experimental period.

\begin{tabular}{lcccccc}
\hline Mes & $\begin{array}{c}\text { Tmax. } \\
\left({ }^{\circ} \mathrm{C}\right)\end{array}$ & $\begin{array}{c}\text { Tmin. } \\
\left({ }^{\circ} \mathrm{C}\right)\end{array}$ & $\begin{array}{c}\text { Tmed. } \\
\left({ }^{\circ} \mathrm{C}\right)\end{array}$ & $\begin{array}{c}\text { HR } \\
(\%)\end{array}$ & $\begin{array}{c}\text { Precipitación } \\
(\mathrm{mm})\end{array}$ & ND \\
\hline Mayo & 26,1 & 14,2 & 19,1 & 74,7 & 73,1 & 4 \\
Junio & 27,0 & 14,0 & 19,4 & 74,3 & 11,3 & 4 \\
Julio & 28,2 & 12,3 & 19,1 & 57,7 & 0,0 & 0 \\
\hline Media & 27,1 & 13,5 & 19,2 & 68,9 & - & - \\
\hline
\end{tabular}

Tmax: temperatura máxima; Tmin: temperatura mínima; Tmed: temperatura media; HR: humedad relativa del aire; ND: número de días con lluvia. Tmax: maximum temperature; Tmin: minimum temperature; Tmed: mean temperature; HR: relative humidity; ND: number of days with rain.

El suelo del área experimental es clasificado como Oxisol (Latossolo Vermelho eutroférrico de acuerdo Embrapa, 2006), que presenta 615, 132 y $253 \mathrm{~g} \mathrm{~kg}^{-1}$ de arcilla, limo y arena, respectivamente. El análisis químico del suelo, a una profundidad de 0 a $20 \mathrm{~cm}$, realizada previamente a la instalación del experimento presentó $136 \mathrm{mg} \mathrm{dm}^{-3}$ de $\mathrm{P}$, lo que corresponde a un valor muy alto, según Trani et al. 
(1997). El suelo presentó también pH 5,8; $24 \mathrm{~g} \mathrm{dm}^{-3} \mathrm{de}$ materia orgánica; 3,4; 43; 37; 83 y $106 \mathrm{mmol}_{\mathrm{c}} \mathrm{dm}^{-3} \mathrm{de}$ $\mathrm{K}, \mathrm{Ca}, \mathrm{Mg}$, suma de bases y capacidad de intercambio catiónico $(\mathrm{CIC})$ respectivamente, y 79\% de saturación por bases.

\section{Diseño experimental y tratamientos}

Fueron evaluados los factores Cultivar: Amanda (grupo de hojas crespas y sueltas); Karla (grupo de hojas lisas y sueltas) y Lucy Brown (grupo de hojas crespas con formación de cabeza) y dosis de $\mathrm{P}\left(0,50,100,200\right.$ y $300 \mathrm{~kg}^{-1}$ de $\left.\mathrm{P}_{2} \mathrm{O}_{5}\right)$, usando como fuente superfosfato triple. Las dosis de fósforo propuestas fueron basadas en la dosis de $200 \mathrm{~kg} \mathrm{ha}^{-1}$ de $\mathrm{P}_{2} \mathrm{O}_{5}$, recomendada por Trani et al. (1997), para el cultivo de lechuga, cuando el contenido de $\mathrm{P}$ en el suelo es muy alto, es decir, superior a $60 \mathrm{mg} \mathrm{dm}^{-3}$ de $\mathrm{P}$.

El diseño experimental fue de bloques aleatorizados, en arreglo factorial de $3 \times 5$, con cuatro repeticiones. El área de la unidad experimental fue de $1,00 \mathrm{~m}$ de ancho por $1,75 \mathrm{~m}$ de largo, y contenía cuatro líneas con siete plantas por línea, es decir, un espaciamiento entre plantas de 0,25 $\mathrm{m}$ y entre líneas de $0,25 \mathrm{~m}$, dando un total de 28 plantas por unidad experimental. Las plantas localizadas en el inicio y final de cada línea, así como las líneas laterales, fueron consideradas como bordadura.

El cultivar Karla tiene un ciclo de 55 a 60 días. Las plantas son grandes y uniformes, adaptadas para ser cultivadas durante todo el año en campo abierto e hidroponía. Presenta buena tolerancia a floración precoz, a la quemadura de los bordes de la hoja ("tip burn") y al virus del mosaico de lechuga (LMV-parotipo II) (Bezerra Neto et al., 2003). El cultivar Amanda presenta ciclo de 55 a 60 días, con plantas grandes y voluminosas, bajo porcentaje de quiebra de hojas, hojas crespas y firmes, las cuales tiene optima apariencia al ser empacadas (Seminis, 2017a). El cultivar Lucy Brown presenta ciclo de 75 días. Las plantas son gruesas dando una protección importante a la cabeza, la cual posee tamaño grande con un corazón pequeño. La coloración es verde clara, con excelente tolerancia a la floración precoz (Seminis, 2017b).

\section{Instalación y conducción del experimento}

Las plántulas fueron producidas en bandejas de polipropileno con 288 alveolos, usándose substrato organomineral Plantmax $\mathrm{HA}^{\circledR}$. La siembra fue realizada el día 5 de mayo de 2008 y el transplante en campo se efectuó cuando las plántulas tenían cuatro hojas.

La preparación del suelo consistió en un pase de arado, rastreo y por último, la conformación de canteros con rotoencanterador. La fertilización al momento de la siembra fue realizada llevando en consideración el análisis del suelo, de acuerdo con la recomendación propuesta por Trani et al. (1997). Fueron utilizados $40 \mathrm{~kg} \mathrm{ha}^{-1}$ de $\mathrm{N}$, en la forma de urea, $50 \mathrm{~kg} \mathrm{ha}^{-1}$ de $\mathrm{K}_{2} \mathrm{O}$, usando como fuente cloruro de potasio y $1 \mathrm{~kg} \mathrm{ha}^{-1}$ de $\mathrm{B}$, utilizándose como fuente ácido bórico, vía pulverización al suelo, siendo incorporados con rotoencanterador. Con relación a las doses de fósforo evaluadas en el presente experimento, estas fueron aplicadas también en el momento de la siembra, de forma no localizada (al voleo). No se aplicaron correctivos en el suelo, debido a que la saturación por bases era del $79 \%$, valor muy cercano al considerado por Trani et al. (1997) como ideal, que es del $80 \%$. La fertilización posterior a la siembra fue realizada con urea, aplicándose $90 \mathrm{~kg} \mathrm{ha}^{-1}$ de $\mathrm{N}$, parcelado a los 10,20 y 30 días después de transplante (DDT) para los tres cultivares, siguiendo las recomendaciones de Trani et al. (1997). Para el cultivar 'Lucy Brown', fue incrementada cada parcela con $30 \mathrm{~kg} \mathrm{ha}^{-1}$ de $\mathrm{N}$, a los 40 DDT, debido a que su ciclo de desarrollo es más extenso.

Las cosechas fueron realizadas el día 23 de julio de 2008 para 'Amanda' y 'Karla' y el día 31 de julio para 'Lucy Brown', completándose de esa manera, un total de 47 días desde el transplante hasta la cosecha para 'Amanda' y 'Karla' y de 55 días para 'Lucy Brown'. La cosecha fue establecida cuando el $80 \%$ de las plantas del área útil de cada parcela se encontraban en el punto de cosecha.

\section{Características evaluadas en el experimento}

Concentración foliar de P: cuando las plantas completaron aproximadamente dos tercios del ciclo productivo (23 DDT para 'Amanda' y 'Karla', y 26 DDT para 'Lucy Brown'), fue realizado un muestreo de hojas recién desarrolladas, siguiendo la recomendación de Trani \& Raij (1997), para la evaluación del estado nutricional. La colecta se realizó en el inicio del día entre las seis y siete horas. Después de la colecta, las hojas fueron llevadas al laboratorio y lavadas con agua corriente y agua desionizada. En seguida, se removió el exceso de agua con papel absorbente, y las muestras fueron colocadas en bolsas de papel, identificadas y llevadas para secar en estufa con circulación forzada de aire, a $65^{\circ} \mathrm{C}$, hasta alcanzar masa constante. Posteriormente, cada muestra fue molida en un molino tipo Wiley. La preparación del extracto para la lectura de concentración de $\mathrm{P}$ fue realizado conforme a Bataglia et al. (1983).

Masa fresca de la parte aérea (MFPA): las plantas fueron pesadas luego de la cosecha, realizada en el inicio de la mañana, entre la seis y siete horas. Fueron seleccionadas tres plantas centrales de cada parcela, dentro del área útil, cortándose el tallo a ras del suelo. Las hojas muertas y senescentes fueron descartadas y la masa determinada con el auxilio de una balanza con sensibilidad de 0,01 g. Para 'Lucy Brown', la MFPA correspondió a la "cabeza" y hojas externas a esta.

Numero de hojas por planta $(\mathrm{NH})$ : en las mismas plantas colectadas para la determinación de la MFPA fueron contadas las hojas.

Área foliar (AF): El área foliar fue evaluada en el medidor electrónico de área foliar marca LI-COR, 
modelo 3100 , en las mismas plantas en las que determinó MFPA.

Masa seca de la parte aérea (MSPA): las hojas y el tallo fueron lavados y colocados para secar en estufa con circulación forzada, a $65^{\circ} \mathrm{C}$, hasta alcanzar masa constante. Después del secado, se realizó el pesaje en balanza electrónica de precisión, con sensibilidad de $0,01 \mathrm{~g}$.

Contenido de $\mathrm{P}$ en el suelo: después de finalizar el experimento fueron colectadas muestras de suelo de cada parcela, en la camada de 0 a $20 \mathrm{~cm}$, para la determinación del contenido de $\mathrm{P}$ disponible. Previamente al muestreo, fue realizada una homogenización del suelo de cada parcela, colectándose ocho sub muestras y colocándolas en un balde, donde posteriormente se mezclaron, tamizaron y eliminaron resto de raíces, conformando de esta forma, una muestra compuesta por parcela. Cada muestra fue secada bajo sombra, tomándose especial cuidado en lavar los implementos utilizados para la colecta de muestras así como los baldes, evitando de esta forma, la contaminación con suelo proveniente de otras par- celas. En seguida fueron llevadas para el laboratorio para la determinación de $\mathrm{P}$, siguiendo la metodología descrita por Raij et al. (2001).

\section{Análisis estadística}

Los datos colectados fueron sometidos a un análisis de variancia por el test $F$, de acuerdo al diseño experimental propuesto. Cuando se encontró efecto significativo de las dosis, se realizó un análisis de regresión, escogiendo la ecuación significativa y con mayor coeficiente de determinación. Para efecto significativo del cultivar, se aplicó el test de Tukey.

\section{Resultados y discusión}

En la tabla 2 se encuentran los valores $F$, coeficientes de variación y significancia de los factores cultivar y dosis de $\mathrm{P}$ sobre las características concentración foliar de $P$, masa fresca de la parte aérea, número de hojas, área foliar, masa seca de la parte aérea y contenido de fósforo en el suelo.

Tabla 2- Análisis de varianza (test $F$ ) para concentración foliar de $P(P F)$, masa fresca de la parte aérea (MFPA), número de hojas (NH), área foliar (AF), masa seca de la parte aérea (MSPA) y contenido de $P$ en el suelo (PS), después de la cosecha de lechuga. Analysis of variance ( $F$ test) on the phosphorus content in leave (PF); fresh weight of the plant aerial part of lettuce (MFPA), number of leaves (NH), leaf area (AF), dry weight of the plant aerial part of lettuce (MSPA) and phosphorus level in soil (PS) after the harvest of lettuce.

\begin{tabular}{lcccccc}
\hline Causas de & PF & MFPA & NH & AF & MSPA & PS \\
Variación & & $118,23^{\star *}$ & $97,50^{*}$ & $20,1^{* *}$ & $3,36^{*}$ & $5,71^{\star *}$ \\
\hline Cultivar (C) & $73,94^{\star *}$ & $3,24^{\star *}$ & $2,38^{\text {ns }}$ & $4,75^{*}$ & $5,52^{* *}$ & $3,08^{*}$ \\
Dosis P (P) & $6,47^{*}$ & $0,93^{\text {ns }}$ & $1,15^{\text {ns }}$ & $0,56^{\text {ns }}$ & $0,53^{\text {ns }}$ & $0,68^{\text {ns }}$ \\
\hline C X P & $2,47^{\star *}$ & 29,6 & 15,3 & 30,9 & 23,5 & 24,1 \\
\hline CV $(\%)$ & 23,3 & 29,6 & & \\
\hline
\end{tabular}

$\left({ }^{\mathrm{ns}}\right)$ no significativo; $\left({ }^{*}\right)$ significativo a $5 \%$ de probabilidad; $\left({ }^{* \star}\right)$ significativo a $1 \%$ de probabilidad por el test $\mathrm{F}$. $\left({ }^{n s}\right)$ non significant; $\left({ }^{*}\right)$ significant at the $5 \%$ level of probability; $\left({ }^{\star *}\right)$ significant at the $1 \%$ level of probability, according to the $F$ test.

Se encontró interacción significativa entre los factores cultivar y dosis de fósforo para la concentración foliar de P (Tabla 2). Los valores de fósforo en las hojas del cultivar 'Lucy Brown' y 'Karla', en función de las dosis de $\mathrm{P}$, se ajustaron a una ecuación linear y la aplicación de dosis crecientes de P promovió incrementos proporcionales en la concentración foliar de $\mathrm{P}$ (Figura 1). Este resultado concuerda con lo verificado por Coutinho et al. (2008) y Bertossi et al. (2013), evaluando la respuesta a fertilización fosfatada de lechuga de los grupos americana y lisa en suelos pobres del nutriente. Los valores de $\mathrm{P}$ en la hoja diagnosticada de la lechuga 'Karla' y 'Lucy Brown', aun con la dosis máxima aplicada (300 kg ha-1 de $\mathrm{P}_{2} \mathrm{O}_{5}$ ), estuvieran por debajo del rango de valores considerados por Trani \& Raij (1997) como adecuados, que es de 4 a $7 \mathrm{~g} \mathrm{~kg}^{-1}$. La concentración foliar de P en 'Karla' fue mayor que la observada para 'Lucy Brown'. Para 'Amanda' hubo ajuste cuadrático a la aplicación de fósforo (Figura 1), encontrando que dosis mayores a $50 \mathrm{~kg} \mathrm{ha}^{-1}$ de $\mathrm{P}_{2} \mathrm{O}_{5}$ proporcionaron concentraciones foliares de $\mathrm{P}$ dentro del rango adecuado, alcanzando en la dosis de $164 \mathrm{~kg} \mathrm{ha}^{-1}$ de $\mathrm{P}_{2} \mathrm{O}_{5}, 4,8 \mathrm{~g} \mathrm{~kg}^{-1}$ de $\mathrm{P}$ foliar. Apartir de este punto, la concentración foliar de $\mathrm{P}$ disminuyó, determinándose con la dosis de $300 \mathrm{~kg}$ ha $^{-1}$ de $\mathrm{P}_{2} \mathrm{O}_{5}$, concentraciones de $\mathrm{P}$ semejantes a las plantas sin aplicación de fósforo, como consecuencia probablemente, de un efecto de dilución en función de un mayor crecimiento, excediendo la tasa de absorción del nutriente, efecto también reportado por Silva et al. (2016) en lechuga 'Vera' y Souza et al. (2014) en sandia, respectivamente, trabajando con altas dosis de $P$.

Se verificó efecto significativo de los factores cultivar y dosis de $\mathrm{P}$, individualmente, sobre la masa fresca de la parte aérea (MFPA) (Tabla 2). La mayor MFPA fue obtenida por 'Lucy Brown', la cual presentó

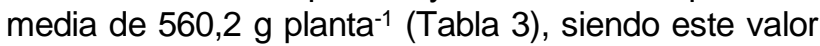
cerca de tres veces mayor que los observados para 'Amanda' y 'Karla'. Según Mota et al. (2003), esto puede deberse al hecho de que la lechuga americana presenta un ciclo mayor en relación a lechugas de los grupos Crespa y Lisa. 


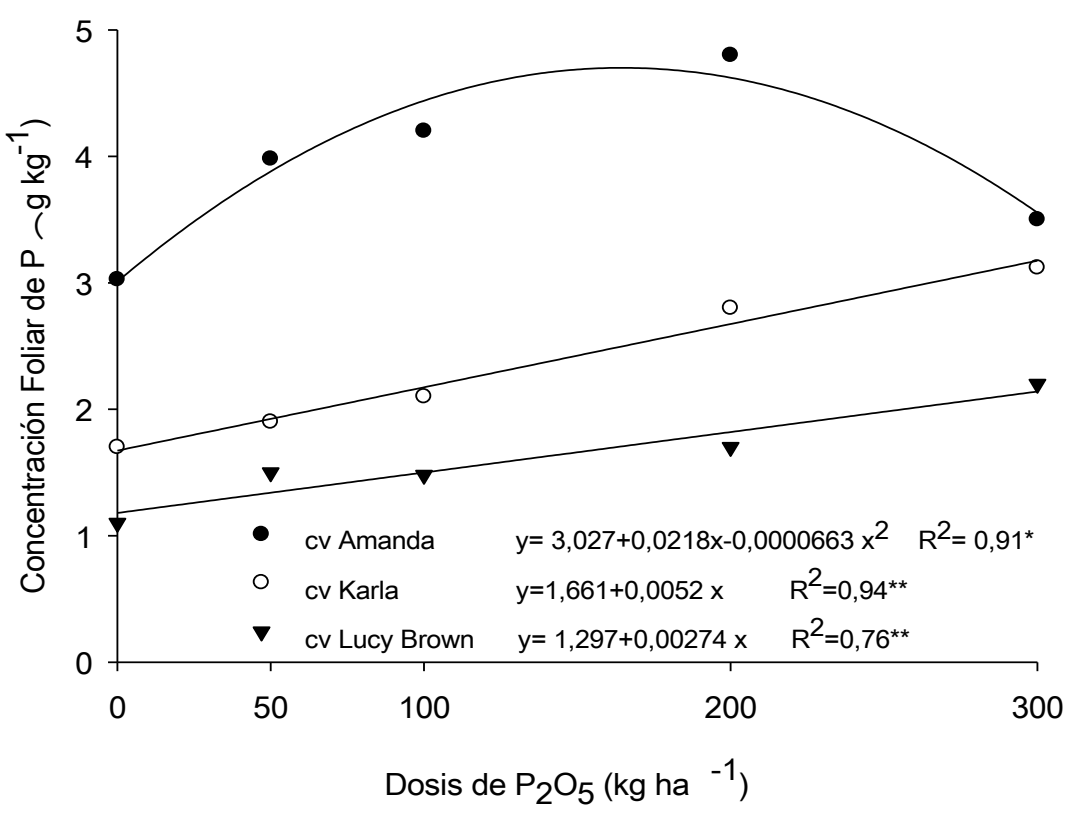

Figura 1 - Concentración de fósforo en hojas de lechuga en función del cultivar y la dosis de fósforo. Phosphorus leaf content in lettuce as influenced by the cultivars and rates of phosphorus.

Tabla 3 - Medias de masa fresca de la parte aérea (MFPA), número de hojas (NH), área foliar (AF), masa seca de la parte aérea (MSPA) y contenido de fósforo en el suelo (PS), de cultivares de lechuga. Means of fresh weight of the plant aerial part (MFPA), number of leaves (NH), leaf area (AF), dry weight of the plant aerial part, and phosphorus level in soil (PS) as influenced by the cultivars of lettuce.

\begin{tabular}{lccccc}
\hline Cultivar & $\begin{array}{c}\text { MFPA } \\
\left(\text { g planta }^{-1}\right)\end{array}$ & $\begin{array}{c}\text { NH } \\
\left(\text { hojas planta }^{-1}\right)\end{array}$ & $\begin{array}{c}\text { AF } \\
\left(\mathrm{cm}^{2}\right)\end{array}$ & $\begin{array}{c}\text { MSPA } \\
\left(\mathrm{g} \mathrm{planta}^{-1}\right)\end{array}$ & $\begin{array}{c}\text { PS } \\
\left(\mathrm{g} \mathrm{kg}^{-1}\right)\end{array}$ \\
\hline Amanda & $208,58^{\mathrm{B}}$ & $24,3^{\mathrm{B}}$ & $2235,6^{\mathrm{C}}$ & $21,53^{\mathrm{AB}}$ & $100,65^{\mathrm{B}}$ \\
Karla & $152,44^{\mathrm{B}}$ & $38,0^{\mathrm{A}}$ & $3430,2^{\mathrm{B}}$ & $20,24^{\mathrm{B}}$ & $100,15^{\mathrm{B}}$ \\
Lucy Brown & $560,20^{\mathrm{A}}$ & $20,2^{\mathrm{C}}$ & $4281,2^{\mathrm{A}}$ & $24,40^{\mathrm{A}}$ & $124,60^{\mathrm{A}}$ \\
\hline
\end{tabular}

*Medias seguidas por la misma letra, en la misma columna, no difieren entre sí por el test de Tukey a $5 \%$ de probabilidad. Means followed by the same letter, in the same column, are not statistically different at the $5 \%$ level of probability, according to Tukey's test.

La MFPA aumentó a medida que mayores dosis de fósforo fueron aplicadas (Figura 2), resultado semejante a los obtenidos por AlKhader \& Abu Rayyan (2015), Chagas et al. (2015) y Silva et al. (2016), en los cultivares Robinson, Solaris y Vera, respectivamente. De acuerdo a Raij (1991), contenidos adecuados de $\mathrm{P}$ en el suelo pueden influenciar la producción de masa fresca en diferentes cultivos, ya que el fósforo estimula el desarrollo de raíces, favoreciendo además, la absorción de otros nutrientes por la planta.

Para número de hojas, hubo efecto significativo apenas del factor cultivar (Tabla 2). 'Karla' del grupo Lisa, fue la que obtuvo mayor número de hojas, 38 hojas planta-1, superando a 'Amanda' y 'Lucy Brown', con medias de 24,3 y 20,2 hojas planta-1, res- $^{-}$ pectivamente (Tabla 3). El número de hojas observado en 'Amanda', del grupo Crespa, se encuentra muy cercano a los valores reportados por Kano et al. (2012) y Gualberto et al. (2008), que fueron de 24 y 20 hojas planta $^{-1}$, respectivamente, para el cultivar Veronica, del mismo grupo Crespa. Hubo ajuste cuadrático de las medias de número de hojas en función de las dosis aplicadas de $\mathrm{P}$ (Figura 3). La dosis de $161 \mathrm{~kg} \mathrm{ha}^{-1}$ de $\mathrm{P}_{2} \mathrm{O}_{5}$ fue la que proporcionó mayor número de hojas. De acuerdo con Raij (1991), el fósforo además de estimular el desarrollo radicular, presenta alta movilidad dentro de la planta, transfiriéndose rápidamente de los tejidos viejos para regiones de meristemos activos, proporcionando mayor actividad celular y consecuentemente, aumento del área foliar y del número de hojas.

Para área foliar, se encontró efecto significativo de los factores cultivar y dosis (Tabla 2). 'Lucy Brown' fue la que presentó mayor área foliar, con una media de $4.3 \mathrm{~cm}^{2}$ planta-1, superando a 'Karla' y 'Amanda', que presentaron 3.4 y $2.2 \mathrm{~cm}^{2}$ planta-1, $^{-1}$ respectivamente (Tabla 3 ). Los valores observados para 'Amanda' fueron menores que los reportados por Feltrim et al. (2009) y Kano et al. (2012), que obtuvieron 4.9 y $2.7 \mathrm{~cm}^{2}$ planta-1 ${ }^{-1}$, respectivamente, en el cultivar Veronica, también del grupo Crespa. El área foliar se ajustó a una ecuación polinómica cuadrática (Figura 3 ), con incrementos positivos hasta la dosis de $203 \mathrm{~kg} \mathrm{ha}^{-1}$ de $\mathrm{P}_{2} \mathrm{O}_{5}$. Kano et al. (2012) verificaron 
respuesta para lechuga crespa a dosis de fósforo, constatando incremento de $1.1 \mathrm{~cm}^{2}$ planta-1 cuando no fue aplicado fósforo, hasta $2.7 \mathrm{~cm}^{2}$ planta-1 con una dosis de $600 \mathrm{~kg} \mathrm{ha}^{-1}$ y que a partir de este valor hasta $800 \mathrm{~kg} \mathrm{ha}^{-1}$ de $\mathrm{P}_{2} \mathrm{O}_{5}$ no se presentaban diferencias significativas para esta variable.

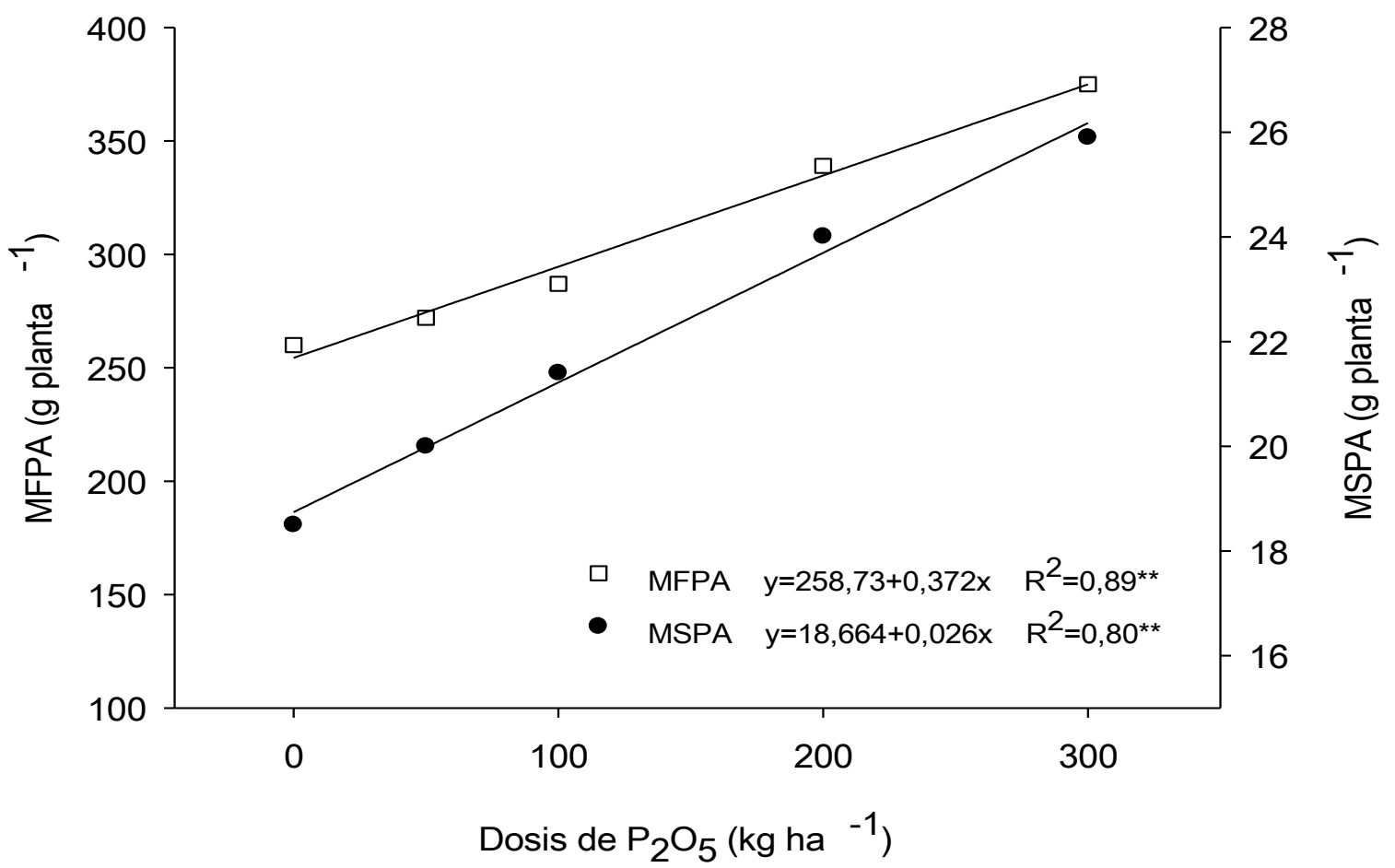

Figura 2 - Masa fresca de la parte aérea (MFPA) y masa seca de la parte aérea (MSPA) de cultivares de lechuga en función de las dosis de fósforo. Fresh weight of the plant aerial part (MFPA) and dry weight of the plant aerial part (MSPA) of cultivars of lettuce as influenced by phosphorus rates.

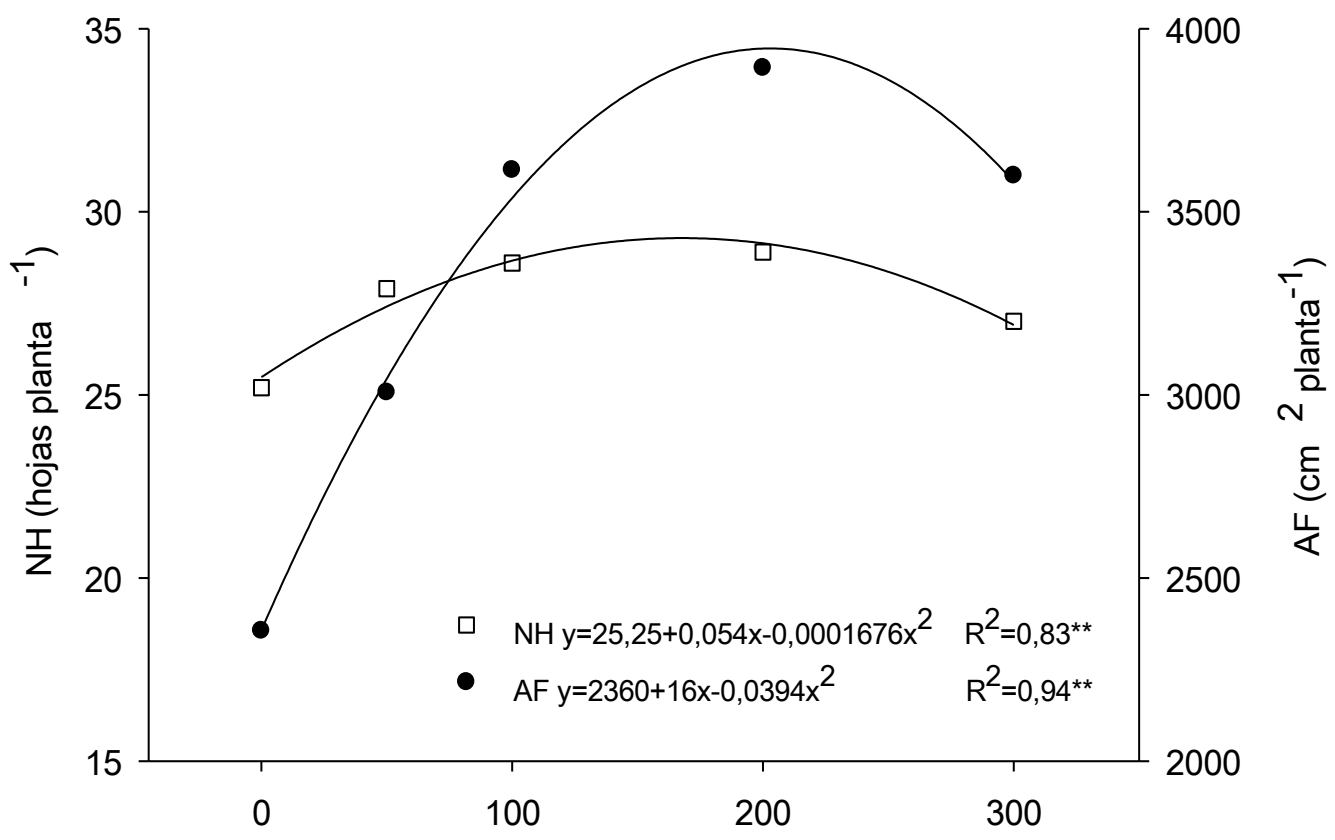

Dosis de $\mathrm{P}_{2} \mathrm{O}_{5}\left(\mathrm{~kg} \mathrm{ha}^{-1}\right)$

Figura 3 - Número de hojas $(\mathrm{NH})$ y área foliar (AF) de cultivares de lechuga en función de las dosis de fósforo aplicadas. Number of leaves $(\mathrm{NH})$ and leaf area $(\mathrm{AF})$ of cultivars of lettuce as influenced by phosphorus rates. 
La MSPA fue influenciada, así como la MFPA, por los dos factores (Tabla 2). 'Lucy Brown' presentó mayor MSPA con una media de 24,4 $\mathrm{g}$ planta $^{-1}$, sin embargo, no se determinó diferencia significativa con el cultivar 'Amanda', que obtuvo una media de 21,5 g planta-1 $^{-1}$ (Tabla 3). De la misma forma que ocurrió para la MFPA, para MSPA también se registraron incrementos con el aumento de las dosis de fósforo aplicadas, ajustándose los valores a una ecuación linear (Figura 2). La MSPA varió de $16 \mathrm{~g}_{\text {planta- }}{ }^{-1}$ en la dosis de $0 \mathrm{~kg} \mathrm{ha}^{-1}$ de $\mathrm{P}_{2} \mathrm{O}_{5}$ hasta 25 g planta $^{-1}$ con la dosis de $300 \mathrm{~kg} \mathrm{ha}^{-1}$ de $\mathrm{P}_{2} \mathrm{O}_{5}$. Estos valores son superiores a los reportados por Grangeiro et al. (2006), Kano et al. (2012) y Mantovani et al. (2014), que encontraron para el cultivar Veronica, valores de $10 ; 11,4$ y 20,0 g planta $^{-1}$, respectivamente. Silva et al. (2016), para el cultivar Vera, también Crespa, encontraron la mayor MSPA con una dosis de $238 \mathrm{~kg} \mathrm{ha}^{-1}$ de $\mathrm{P}_{2} \mathrm{O}_{5}$.

Los resultados confirman que la lechuga es responsiva a la fertilización con $\mathrm{P}$, aun en suelos con altos contenidos del nutriente. Los tres cultivares trabajados respondieron positivamente en producción de MFPA y MSPA a la aplicación de hasta $300 \mathrm{~kg} \mathrm{ha}^{-1}$. Esta dosis es mayor de la recomendada $\left(200 \mathrm{~kg} \mathrm{ha}^{-1}\right.$ de $\mathrm{P}_{2} \mathrm{O}_{5}$ ) por Trani et al. (1997), cuando el contenido de $\mathrm{P}$ en el suelo es considerado alto. Cecílio Filho et al. (2013) también encontraron en el cultivo de repollo en un Oxisol muy arcilloso con alto contenido de P, mayores rendimientos con dosis superiores a las recomendadas por Trani et al. (1997). Cabe destacar que los fertilizantes fosfatados por ser susceptibles a reacciones de adsorción y precipitación en suelos tropicales de textura arcillosa, para una adecuada disponibilidad de $\mathrm{P}$ en las plantas es necesario la aplicación de altas dosis del nutriente al suelo (Bull et al., 2004).

Para el contenido de $\mathrm{P}$ en el suelo, se observó efecto significativo para los factores cultivar y dosis (Tabla 2). Mayor contenido de $\mathrm{P}$ en el suelo fue encontrado en las parcelas cultivadas con 'Lucy Brown' (Tabla 3). Hubo ajuste significativo linear para el aumento de la dosis y el contenido de $\mathrm{P}$ en el suelo (Figura 4), determinándose incremento del $32 \%$ en el contenido de $\mathrm{P}$ en el suelo con la dosis de $300 \mathrm{~kg} \mathrm{ha}^{-1}$ de $\mathrm{P}_{2} \mathrm{O}_{5}$, en comparación al control (sin aplicación). Según Costa et al. (2006), en razón del factor de capacidad de fósforo de los suelos de textura arcillosa, solo se consiguen alteraciones en el contenido de $P$ en el suelo con dosis muy elevadas o con la aplicación de cal durante largos periodos de tiempo (Verma et al., 2005). Schaefer et al. (2004), destacan que en suelos oxisoles puede ocurrir alta adsorción de fósforo por los coloides amorfos y por la materia orgánica, limitando la disponibilidad por las plantas, aun cuando se aplican altas doses del nutriente. Por otro lado, adiciones de fósforo en suelos ácidos o moderadamente ácidos, suelen tener poco efecto sobre los contenidos de $\mathrm{P}$ soluble, debido a que en estas condiciones, los óxidos de hierro y aluminio presentan preferencialmente cargas positivas, reteniendo en su superficie varios aniones, entre los que se encuentran los iones fosfatos, (Verma et al., 2005).

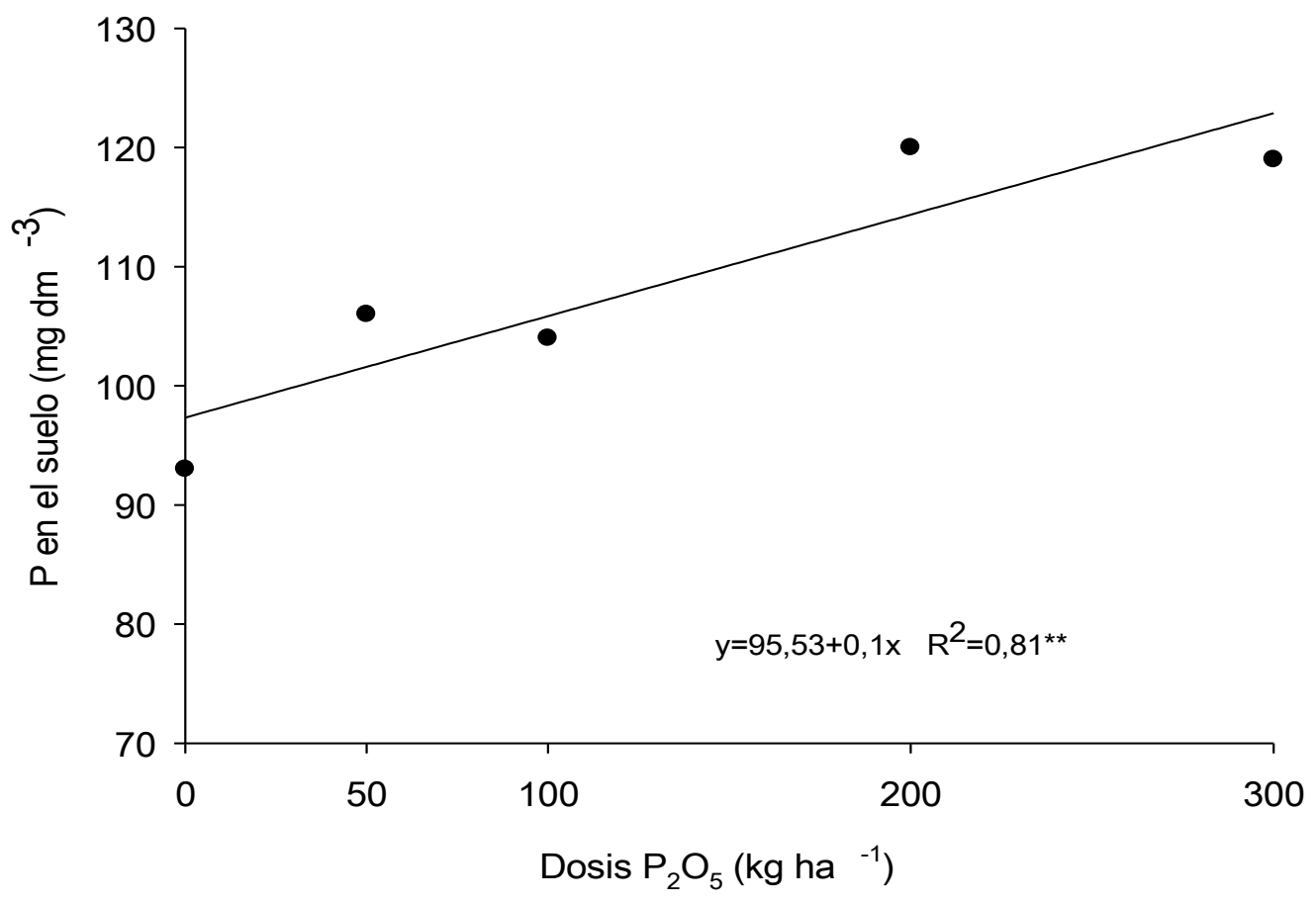

Figura 4 - Contenido de fósforo (PS) en el suelo en función de las dosis del nutriente aplicado. Phosphorus soil content (PS) as influenced by phosphorus rates. 
El contenido de fósforo en el suelo después del experimento varió de 95,5 a $125,5 \mathrm{mg} \mathrm{dm}^{-3}$ (Figura 4), condición que ya fue descrita por Castellane et al. (1988) reportando que áreas con producción intensiva presentan, frecuentemente, contenido de fósforo en el suelo mayor a $100 \mathrm{mg} \mathrm{dm}^{-3}$. Ciertamente, contenidos altos y muy altos de $\mathrm{P}$ en suelo, de acuerdo a la clasificación de Raij et al. (1997), pueden traer consecuencias negativas al medio ambiente si no se toman los cuidados necesarios para la conservación del suelo evitando la erosión y eutrofización de ríos, lagunas y cuerpos de agua.

\section{Conclusiones}

Los cultivares 'Amanda', 'Karla' y 'Lucy Brown' muestran respuesta semejante y positiva al aumento de la disponibilidad de fósforo en el suelo, aun en un Oxisol con contenido muy alto de fósforo $\left(136 \mathrm{mg} \mathrm{dm}^{3}\right)$.

\section{Referencias}

Alkhader AM, Abu-Rayyan AM (2015) The impact of phosphorus fertilizers on nutrients content and uptake in lettuce (Lactuca sativa L.) and on selected chemical properties of a calcareous soil. Jordan Journal of Agricultural Sciences 11(4):1021-1035.

Bataglia OC, Furlani AM, Teixeira JP, Furlani PR, Gallo JR (1983) Métodos de análise química de plantas. Campinas: Instituto Agronômico, 48p.

Batista MA, Vieira AL, De Souza PJ, De Freitas JD, Bezerra Neto $F$ (2012) Efeito de diferentes fontes de adubação sobre a produção de alface no município de Iguatu-CE. Revista Caatinga 25(3): 8-11.

Bertossi AP, Thomazini A, Fonseca AS, Amaral JF (2013) Nutritional efficiency of phosphorus in lettuce. Journal of Agricultural Science 5(8):125-131.

Bezerra Neto F, Andrade FV, Negreiros MZ, Santos Junior JS (2003) Desempenho agroeconômico do consorcio cenoura $x$ alface lisa em dois sistemas de cultivo em faixa. Horticultura Brasileira 21(4):635-641.

Bull LT, Costa MC, Novello A, Fernandes DM, Boas $\mathrm{RL}$ (2004) Doses and forms of application of phosphorus in vernalized garlic. Scientia Agricola 61:516-521. doi:10.1590/S0103-90162004000500009

Castellane PD, Ferreira ME, Maeda AH (1988) Diagnose da fertilidade dos solos cultivados com olerícolas em Atibaia (SP). Horticultura Brasileira 6(2):50.

Cecílio Filho AB, Silva GS, Mendoza-Cortez JW (2013) Phosphorus fertilization of 'Fuyutoyo' cabbages in phosphorus-rich Eutrustox soil. Chilean Journal of Agricultural Research 73(3):288-292. doi:10.4067/S0718-58392013000300012
Cecilio Filho AB, Bonela GD, Da Cruz MC, MendozaCortez JW, Toscano CR (2015) Potassium fertilization of lettuce in potassium-rich Eutrudox soil. African Journal of Agricultural Research 10(4):194-199. doi:10.5897/AJAR2014.8791

Chagas WF, Emrich EB, Guelfi DR, Caputo AL, Faquin V (2015) Características produtivas, nutrição e eficiência agronômica do MAP revestido com polímeros em cultivos de alface. Revista Ciência Agronômica 46(2):266-276. doi:10.5935/1806-6690.20150006

Costa JP, Barros NF, Albuquerque AW, Moura Filho G, Santos JR (2006) Fluxo difusivo de fósforo de doses e da umidade do solo. Revista Brasileira de Engenharia Agrícola e Ambiental 10(4):828-835. doi: 10.1590/S1415-43662006000400007

Coutinho EL, Carvalho FP, Franco HC, Oriolo Júnior V, Coutinho Neto AM, Ueta FZ (2008) Adubação fosfatada em cultivares de alface cultivada em solos defi-

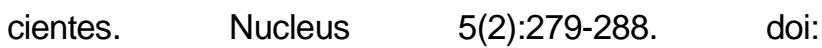
10.3738/nucleus.v5i2.87

EMBRAPA (2006) Empresa Brasileira de Pesquisa Agropecuária. Sistema brasileiro de classificação de solos. Rio de Janeiro: Embrapa Solos. 286p.

Feltrim AL, Cecílio Filho AB, Rezende BL, Branco RB (2009) Produção de alface crespa em solo e em hidroponia, no inverno e verão, em Jaboticabal-SP. Científica 37(01):9-15. doi:10.15361/1984$5529.2009 v 37 n 1 p 9+-+15$

Grangeiro LC, Da Costa KR, Medeiros MA, Salviano AM, Negreiros MZ, Bezerra Neto F, Oliveira SL (2006) Acúmulo de nutrientes por três cultivares de alface cultivadas em condições do semi-árido. Horticultura Brasileira 24(2):190-194. doi:10.1590/S010205362006000200013

Gualberto R, Oliveira PR, Guimaraes AM (2008) Avaliação de cultivares de alface crespas e lisas, em diferentes épocas de cultivos no sistema hidropônico 'NFT'. Nucleus 5(2):341-352. doi:10.3738/nucleus.v5i2.88

Jiao X, Chen W, Chang AC, Page AL (2012) Environmental risk of trace elements associated with long-term phosphate fertilizers applications: a review. Environmnetal Pollution 168:44-52. doi:10.1016/j.envpol.2012.03.052

Jordan-Meille L, Rubaek GH, Ehlert PA, Genot V, Hofman G, Goulding K, Recknagel J, Provolo G, Barraclough $P$ (2012) An overview of fertilizer-P recommendations in Europe: soil testing, calibration and fertilizer recommendations. Soil Use and Management 28(4):419-435. doi:10.1111/j.1475-2743.2012.00453.x 
Kano C, Cardoso Al, Villas Boas RL (2012) Acumulo de nutrientes e resposta da alface a adubação fosfatada. Biotemas 25(3):39-47. doi:10.5007/21757925.2012v25n3p39

Mantovani JR, Oliveira IA, Marques DJ, Da Silva AB, Landgraf PR (2014) Teores de fósforo no solo e produção de alface crespa em função de adubação fosfatada. Ciências Agrárias 35(4):2369-2380. doi:10.5433/1679-0359.2014v35n4Suplp2369

Mota JH, Yuri JE, Resende GM, Oliveira CM, Souza RJ, Freitas AS, Rodrigues Junior JC (2003) Produção de alface americana em função da aplicação de doses e fontes de fósforo. Horticultura Brasileira 21(4):620622. doi:10.1590/S0102-05362003000400008

Petrazzini LL, Souza GA, Rodas CL, Emrich EB, Carvalho JG, Souza RJ (2014) Nutritional deficiency in crisphead lettuce grown in hydroponics. Horticultura Brasileira 32(3):310-313. doi:10.1590/S010205362014000300012

Raij B V (1991) Fertilidade do solo e adubação. Piracicaba: Potafos. 343p.

Raij BV, Andrade JC, Cantarella H, Quaggio JA (2001) Análise química para avaliação da fertilidade de solos tropicais. Campinas: Instituto Agronômico. 285p.

Raij BV, Cantarella H, Quaggio JÁ, Furlani AM (1997) Recomendações de adubação e calagem para 0 Estado de São Paulo. Campinas: Instituto Agronômico. $285 p$.

Sala FC, Costa CP (2012) Retrospectiva e tendência da alfacicultura brasileira. Horticultura Brasileira 30(2):187-194. doi:10.1590/S0102

\section{2}

Seminis, (2017) Alface Amanda. Disponible en <http://www.seminis.com.br/Produtos/amanda/386> (Acceso en 30 ene 2017).

Seminis, (2017) Alface Lucy Brown. Disponible en <http://www.seminis.com.br/Produtos/lucy-brown/383> (Acceso en 30 ene 2017).
Schaefer CE, Gilkes RJ, Fernandes RB (2004) EDS/SEM study of microaggregates of Brazilian Latosols, in relation to $P$ adsorption and clay fraction attributes. Geoderma 123(2):69-81. doi: 10.1016/j.geoderma.2004.01.033

Silva AS, Cecílio Filho AB, Nascimento SM, Vargas PF (2016) Phosphorus fertilizer for lettuce grown in Ultisol in the state of Maranhão. Científica 44(1):64-70.

Silveira AL, Pizolato Neto A, Oliveira AR, Souza LN, Charlo HC (2015) Doses de fósforo para a produção de alface americana com e sem aplicação foliar de zinco. Revista Biotemas 28(1):31-35. doi:10.5007/2175-7925.2015v28n1p31

Souza MS, Medeiros JF, Silva MV, Silva OM, Chaves SW (2014) Estado nutricional da melancia fertirrigada com doses de nitrogênio e fósforo. Semina: Ciências Agrárias 35(4):2301-2316. doi: 10.5433/16790359.2014v35n4Suplp2301

Tóth G, Guicharnaud RA, Tóth B, Hermann T (2014) Phosphorus levels in croplands of the European Union with implications for $\mathrm{P}$ fertilizer use. European Journal of Agronomy 55:42-52. doi:10.1016/j.eja.2013.12.008

Trani PE, Passos FA, Azevedo Filho JA (1997) Alface, almeirão, chicória, escarola, rúcula e agrião d'água. In: Raij BV, Cantarella H, Quaggio JA, Furlani AM (ed) Recomendações de adubação e calagem para 0 Estado de São Paulo. Campinas: Instituto Agronômico. p.168-170.

Trani PE, Raij BV (1997) Hortaliças. In: Raij BV, Cantarella H, Quaggio JÁ, Furlani AM (ed) Recomendações de adubação e calagem para o Estado de São Paulo. Campinas: Instituto Agronômico. p.155-185.

Verma S, Subehia SK, Sharma SP (2005) Phosphorus fractions in an acid soil continuously fertilized with mineral and organic fertilizers. Biology and Fertility of Soils 41:295-300. 